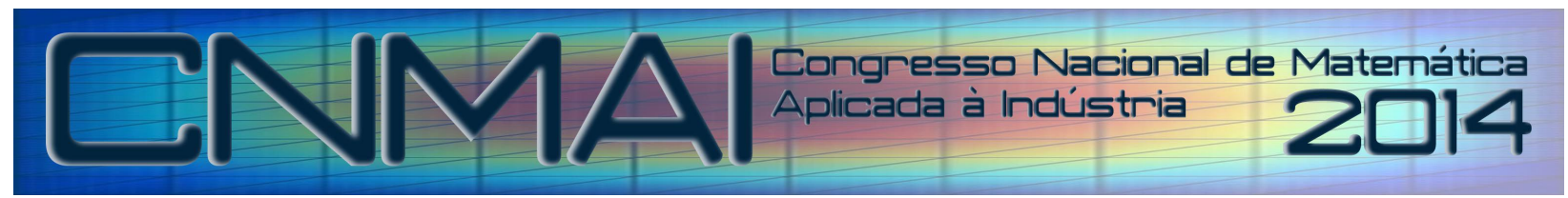

18 a 21 de novembro de 2014, Caldas Novas - Goiás

\title{
MODELAGEM POR ELEMENTOS FINITOS DE ESTRUTURAS COMPOSTAS CONTENDO MATERIAIS POROELÁSTICOS
}

\author{
Antônio Marcos Gonçalves de Lima, amglima@ mecanica.ufu.br \\ Victor Augusto da Costa Silva, Victor_augusto_cn@hotmail.com
}

Universidade Federal de Uberlândia - Faculdade de Engenharia Mecânica, Campus Santa Mônica, Uberlândia-MG Universidade Federal de Uberlândia - Faculdade de Engenharia Mecânica, Campus Santa Mônica, Uberlândia-MG

\begin{abstract}
Resumo: $O$ estudo do comportamento dinâmico de estruturas compostas incorporando materiais poroelásticos para fins de atenuação dos níveis de vibração e ruído é motivado pela sua grande importância tecnológica. Neste contexto, o presente trabalho é dedicado ao estudo numérico-computacional de estruturas compostas do tipo placas finas contendo elementos poroelásticos aplicados sob a forma de tratamentos superficiais na configuração de elementos multicamadas. Para a modelagem do comportamento dinâmico dos materiais poroelásticos, será utilizada a teoria generalizada de Biot. Já para a modelagem dos elementos estruturais compostos incorporando os materiais poroelásticos para serem aplicados sob a forma de tratamentos superficiais (camadas livres e restritas), será utilizada a teoria da camada equivalente única combinada com a teoria de cisalhamento de primeira ordem. Os resultados numéricos demonstram o interesse em se utilizar os materiais poroelásticos para o controle passivo de vibrações e ruído de sistemas dinâmicos.
\end{abstract}

Palavras-chave: Estruturas compostas, Materiais poroelásticos, Controle passivo, Elementos finitos

\section{INTRODUÇÃO}

A acirrada competição existente no mercado internacional globalizado impulsiona a modernização de processos e produtos industriais, notadamente no que diz respeito ao conforto e segurança dos operadores e usuários. Além disso, normas ambientais cada vez mais severas impõem limites restritos para os níveis de vibração e ruído de veículos, máquinas e equipamentos industriais, que estão freqüentemente presentes em vários ramos da atividade produtiva, tais como as indústrias de construção civil, automotiva, aeroespacial, naval, de telecomunicações, mineração, metalúrgica, siderúrgica, de geração e distribuição de energia, etc. Neste panorama, a importância dada ao problema de atenuação dos níveis de vibração e ruído de sistemas mecânicos é hoje um dos problemas cruciais da Engenharia Mecânica, em virtude da tendência de realização de estruturas cada vez mais extensas e leves, e de aumento considerável da velocidade de operação das máquinas. Por exemplo, o ruído dentro de aeronaves tem sido a maior causa do desconforto de passageiros e usuários, e este problema tem-se agravado cada vez mais com o uso progressivo de materiais compostos e motores mais potentes, os quais tendem a amplificar os problemas de vibrações e ruído dessas estruturas.

Dentre as diversas estratégias de controle passivo que têm sido freqüentemente empregadas para tratar o problema de atenuação dos níveis de vibração e ruído de sistemas dinâmicos, o uso de materiais viscoelásticos e poroelásticos é uma estratégia bastante interessante para ser investigada, em virtude de sua grande eficiência e do seu baixo custo de manutenção e simplicidade de aplicação (de Lima et al., 2012; Nashif et al., 1985). Além disso, embora eles percam no quesito adaptabilidade, e apesar do grande interesse na aplicação de técnicas de controle ativo e semi-ativo, o uso desses materiais apresenta-se geralmente como sendo a solução mais robusta e econômica (Zwikker e Kosten, 1949; Delany e Bazley, 1970; Nashif et al., 1985; de Lima et al., 2010). Como resultado, tem-se observado nos últimos anos um aumento considerável do uso dos materiais viscoelásticos e poroelásticos em um grande número de sistemas de engenharia tais como automóveis e aeronaves, estruturas de engenharia civil, mecanismos flexíveis, compressores de refrigeração (de Lima et al., 2007), etc.

Dessa forma, em conseqüência da importância dos materiais poroelásticos e do seu emprego cada vez mais frequente, neste trabalho, será apresentado um procedimento de modelagem por elementos finitos de estruturas compostas do tipo placas finas incorporando os materiais poroelásticos para a redução das vibrações e ruído. 


\section{MODELAGEM MECÂNICA DE PLACAS COMPOSTAS}

Os materiais compostos representam a união de dois ou mais materiais diferentes de forma a ter efeitos sinergéticos entre esses materiais que levem a propriedades mecânicas que dificilmente se conseguiria via aplicação dos materiais constituintes de forma isolada (Faria, 2006). Nos materiais compostos, geralmente se distinguem pelo menos duas fases: a matriz e o reforço. A matriz é um elemento contínuo que tem como principal função transmitir os esforços por todo o material, em especial aos elementos de reforço; proteger o reforço de agentes externos como intempéries, ataque químico e desgastes mecânicos como abrasão. Além disso, ela serve de elemento de união (ou aglutinante) entre os reforços. Já os reforços são os principais responsáveis pela resistência do material composto. Nos trabalhos de Reddy (1997) e Mendonça (2005), diversas teorias têm sido propostas para a modelagem de vigas, placas e cascas compostas são encontradas, como a Teoria Clássica dos Laminados (CLT), a Teoria de Deformação Cisalhante de Primeira Ordem (FSDT), e a Teoria de Deformação Cisalhante de Ordem Superior (HSDT). Entretanto, é importante destacar que as teorias CLT e FSDT apresentam erros na predição das tensões em estruturas compostas espessas, onde a relação entre a espessura e o comprimento da placa é maior que 0,25 (Faria, 2006; Mendonça, 2005). Neste caso, estas teorias são comumente recomendadas para a modelagem de estruturas do tipo placas compostas finas e moderadamente finas (Berthelot, 1992). Segundo Reddy (1997), a teoria HSDT fornece melhor previsão das tensões cisalhantes transversais, ou seja, bem mais próximas da solução exata fornecida pela Teoria da Elasticidade Tridimensional, já que considera a deformação cisalhante transversal com distribuição parabólica. Portanto, ela é mais apropriada para a modelagem de placas compostas mais espessas (espessura/comprimento>0,25). Entretanto, segundo Kulkarni e Bajoria (2003) e Mendonça (2005), o alto custo computacional via utilização da teoria HSDT deve ser levado em conta.

Matematicamente, a principal diferença entre as teorias FSDT e HSDT reside na ordem das funções polinomiais escolhidas para aproximar os campos de deslocamentos mecânicos, sendo linear na teoria FSDT e de terceira ordem na teoria HSDT. Considera-se que a teoria FSDT possui uma melhor relação entre capacidade de predição e custo computacional, mas mostra-se inadequada na predição de freqüências naturais e modos de vibração de ordens elevadas e de distribuições das tensões sob o estratificado. Além disso, podem apresentar problemas de travamento comumente conhecido como shear locking na modelagem de estruturas compostas extremamente finas (espessura/comprimento<0,02), além de requerer um fator de correção para as deformações cisalhantes transversais (Mendonça, 2005).

Neste trabalho, o elemento a ser utilizado para a modelagem de placas compostas finas e moderadamente finas é ilustrado na Fig. 1, onde $z_{k}, h_{k}$ e $\theta_{k}$ indicam, respectivamente, a coordenada ao longo da espessura, a espessura e o ângulo de orientação das fibras unidirecionais de uma camada qualquer $k$. Será também empregada a teoria FSDT, no qual os deslocamentos em um ponto qualquer do elemento podem ser expressos da seguinte forma:

$$
\mathbf{U}(x, y, z, t)=\mathbf{A}(z) \hat{\mathbf{u}}(x, y, t)
$$

Na Eq. (1), tem-se:

$$
\begin{gathered}
\mathbf{U}(x, y, z, t)=[u(x, y, z, t) v(x, y, z, t) w(x, y, z, t)]^{T} \\
\hat{\mathbf{u}}(x, y, t)=\left[u_{0}(x, y, t) v_{0}(x, y, t) w_{0}(x, y, t) \psi_{x}(x, y, t) \psi_{y}(x, y, t)\right]^{T} \\
\mathbf{A}(z)=\left[\begin{array}{lllll}
1 & 0 & 0 & z & 0 \\
0 & 1 & 0 & 0 & z \\
0 & 0 & 1 & 0 & 0
\end{array}\right]
\end{gathered}
$$

onde $u(x, y, z, t), v(x, y, z, t) \mathrm{e} w(x, y, z, t)$ são, respectivamente, os deslocamentos nas direções $x, y$ e $z$. $\left(u_{0}, v_{0}, w_{0}\right) \mathrm{e}$ $\left(\psi_{x}, \psi_{y}\right)$ são os deslocamentos no plano médio nas direções $x, y$ e $z$, e as rotações da seção transversal, respectivamente. 


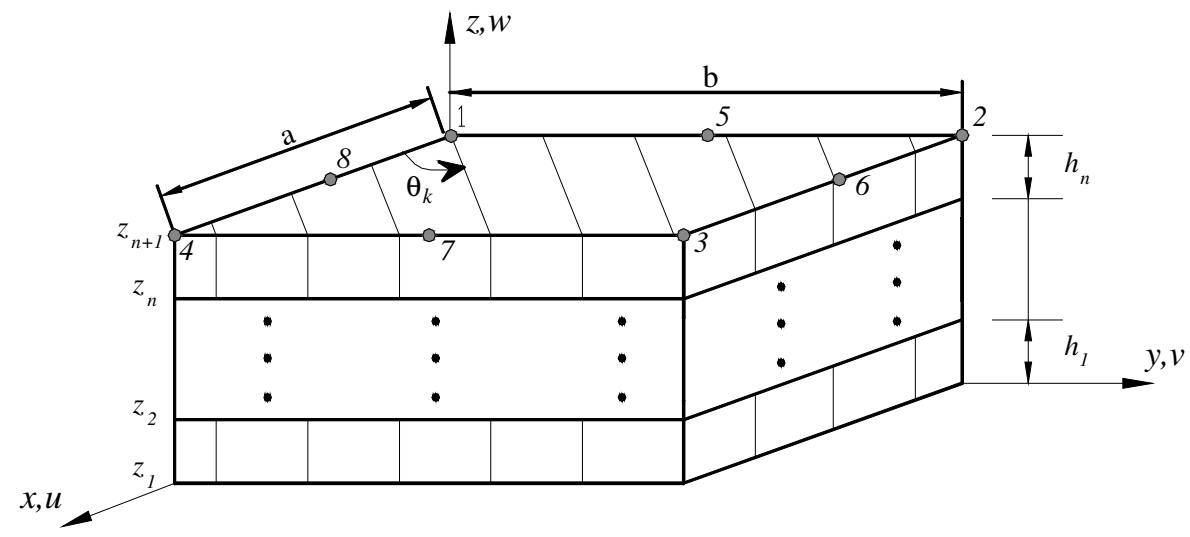

Figura 1. Componentes de um elemento finito de placa plana composta (adaptado de de Lima et al., 2010).

\subsection{Deslocamentos-Deformações e Discretização por Elementos Finitos}

As relações usuais entre as deformações e os deslocamentos são usadas, e as deformações resultantes podem ser calculadas como segue:

$$
\boldsymbol{\varepsilon}(x, y, z, t)=\mathbf{D}(z) \hat{\mathbf{u}}(x, y, t)
$$

onde $\varepsilon(x, y, z, t)=\left[\varepsilon_{x} \varepsilon_{y} \gamma_{x y}\right]^{T}, \quad \varepsilon_{x}=\partial u / \partial x, \quad \varepsilon_{y}=\partial v / \partial y, \quad \gamma_{x y}=\partial u / \partial y+\partial v / \partial x$. A matriz $\mathbf{D}(z)$ é formada pelos operadores diferenciais das relações deformações-deslocamentos.

A discretização do campo de deslocamentos é feita através da utilização de funções de interpolação. Neste caso, para um elemento de placa plana retangular de 8 nós da família serendipity (Reddy, 1997), as cinco variáveis que formam o vetor $\mathbf{u}_{\mathrm{e}}(x, y, t)$ são interpoladas a partir dos seus valores nodais conforme a relação seguinte:

$$
\hat{\mathbf{u}}(x, y, t)=\mathbf{N}(x, y) \mathbf{u}_{\mathrm{e}}(t)
$$

onde $\quad \mathbf{u}_{\mathrm{e}}(t)=\left[\begin{array}{llll}\mathbf{u}_{1}^{T} & \mathbf{u}_{2}^{T} & \ldots & \mathbf{u}_{8}^{T}\end{array}\right]^{T}, \mathbf{u}_{i}^{T}(t)=\left[\begin{array}{lllll}u_{i} & v_{i} & w_{i} & \psi_{x i} & \psi_{y i}\end{array}\right]^{T} \quad(i=1 a 8) . \mathbf{N}(x, y) \in R^{5 x 40}$ é a matriz formada pelas funções de interpolação do elemento da família serendipity formulada no sistema de coordenadas global (de Lima et al., 2010).

As Eqs. (1) e (3) podem ser então definidas em termos das variáveis nodais como segue:

$$
\begin{gathered}
\mathbf{U}(x, y, z, t)=\mathbf{A}(z) \mathbf{N}(x, y) \mathbf{u}_{\mathrm{e}}(t) \\
\boldsymbol{\varepsilon}(x, y, z, t)=\mathbf{D}(z) \mathbf{N}(x, y) \mathbf{u}_{\mathbf{e}}(t)=\mathbf{B}(x, y, z) \mathbf{u}_{\mathrm{e}}(t)
\end{gathered}
$$

Utilizando as interpolações dos deslocamentos e deformações, as expressões das energias cinéticas e de deformação podem ser formuladas, respectivamente, como segue:

$$
T_{E}(t)=\frac{1}{2} \dot{\mathbf{u}}_{\mathrm{e}}^{\mathrm{T}}(t) \mathbf{M}^{(\mathrm{e})} \dot{\mathbf{u}}_{\mathrm{e}}(t), \quad V_{E}(t)=\frac{1}{2} \mathbf{u}_{\mathrm{e}}^{\mathrm{T}}(t) \mathbf{K}^{(\mathrm{e})} \mathbf{u}_{\mathrm{e}}(t)
$$

onde as matrizes elementares de massa e rigidez mecânica são calculadas da seguinte forma:

$$
\begin{aligned}
& \mathbf{M}_{\mathrm{e}}^{(\mathrm{e})}=\sum_{k=1}^{n} \int_{z=z_{k}}^{z_{k+1}} \int_{y=0}^{y=b} \int_{x=0}^{x=a} \rho_{k} \mathbf{N}^{T}(x, y) \mathbf{A}^{T}(z) \mathbf{A}(z) \mathbf{N}(x, y) d x d y d z \\
& \mathbf{K}_{\mathrm{e}}^{(\mathrm{e})}=\sum_{k=1}^{n} \int_{z=z_{k}}^{z_{k+1}} \int_{y=0}^{y=b} \int_{x=0}^{x=a} \mathbf{B}^{T}(x, y, z) \mathbf{C}\left(\theta_{k}\right) \mathbf{B}^{T}(x, y, z) d x d y d z
\end{aligned}
$$

$\mathrm{Na}$ Eq. (7b), $\mathbf{C}\left(\theta_{k}\right)$ é a matriz das propriedades elásticas ortotrópicas, construída de acordo com a teoria CLT via matriz de transformação, conforme detalhado no trabalho de Lima et al. (2010). 
Após a montagem das matrizes elementares, a equação do movimento a nível global pode ser escrita, no domínio do tempo, da seguinte forma:

$$
\mathbf{M}_{\mathrm{e}} \ddot{\mathbf{u}}_{\mathrm{e}}(t)+\mathbf{K}_{\mathrm{e}} \mathbf{u}_{\mathrm{e}}(t)=\mathbf{f}_{\mathrm{e}}(t)
$$

onde $\mathbf{M}_{\mathrm{e}}=\bigcup_{e=1}^{\text {nelem }} \mathbf{M}_{\mathrm{e}}^{(\mathrm{e})}, \mathbf{K}_{\mathrm{s}}=\bigcup_{e=1}^{\text {nelem }} \mathbf{K}_{\mathrm{s}}^{(\mathrm{e})}$ são as matrizes globais de massa e rigidez da estrutura composta, e o símbolo $\bigcup$ indica a montagem matricial. $\mathbf{u}_{\mathrm{e}}(t)$ e $\mathbf{f}_{\mathrm{e}}(t)$ designam, respectivamente, os vetores dos graus de liberdade mecânicos globais e das forças generalizadas. Além disso, através da Eq. (8) é possível calcular as respostas dinâmicas do sistema nos domínios do tempo e da frequência.

\section{MODELAGEM DE MATERIAIS POROELÁSTICOS}

Entre as teorias que descrevem a propagação do som em materiais porosos, o modelo empírico proposto por Delany e Bazley (1970) a partir de medidas experimentais, é um dos mais empregados, devido à sua simplicidade. Ele fornece a impedância característica $Z_{e}$ e a constante de propagação $k_{e}$ do meio fluído, necessitando apenas do conhecimento da resistividade $\sigma$ de passagem do fluxo de ar. Outros modelos do tipo fluído equivalente foram também desenvolvidos (Champoux e Allard, 1991; Zwikker e Kosten, 1949). Entretanto, esses modelos não são adaptados para serem utilizados quando o esqueleto está em movimento, e neste sentido, o modelo generalizado de Biot (1956) baseado em uma descrição macroscópica do deslocamento de duas fases do material, constitui um modelo fundamental para descrever o comportamento dinâmico dos materiais poroelásticos.

O modelo de elementos finitos de materiais poroelásticos é construído diretamente do modelo de Biot em deslocamento. A formulação $\mathbf{u}_{\mathbf{p}}=[\mathbf{u}, \mathbf{U}]^{T}$ que considera o movimento do esqueleto possui basicamente seis graus de liberdade por nó: três translações para a fase sólida contidas no vetor, $\mathbf{u}$, e três translações para a fase fluído formando o vetor, U (Panneton e Atalia, 1996, 1994; Johansen et al., 1995). Entretanto, o estudo da propagação acústica em um meio poroso necessita ainda da consideração das seguintes hipóteses: escala macroscópica do meio poroso; hipótese de que o comprimento de onda deve ser maior que um elemento de volume; pequenos deslocamentos (elasticidade linear); as variáveis que descrevem o movimento possuem dependência temporal da forma, $e^{j \omega t}$, o que caracteriza o regime harmônico. Neste sentido, as expressões para as energias potencial e cinética de um elemento finito poroelástico podem ser escritas como segue (Dauchez et al., 1998a):

$$
\begin{gathered}
V^{(e)}=\frac{1}{2}\left[\mathbf{L}^{T} \mathbf{u}^{T} \mathbf{H}_{s} \mathbf{L u}+2 \operatorname{Re}\left(\mathbf{L}^{T} \mathbf{u}^{T} \mathbf{H}_{s f} \mathbf{L} \mathbf{U}+\mathbf{L}^{T} \mathbf{U}^{T} \mathbf{H}_{f} \mathbf{L} \mathbf{U}\right)\right] \\
T^{(e)}=\frac{1}{2}\left[\rho_{12} \dot{\mathbf{u}}^{T} \dot{\boldsymbol{u}}+2 \rho_{12} \operatorname{Re}\left(\dot{\mathbf{u}}^{T} \dot{\mathbf{U}}+\rho_{12} \dot{\mathbf{U}}^{T} \dot{\mathbf{U}}\right)\right]
\end{gathered}
$$

onde $\mathbf{u}=\left[\begin{array}{lll}u_{x} & u_{y} u_{z}\end{array}\right]^{T}$ e $\mathbf{U}=\left[\begin{array}{lll}U_{x} & U_{y} & U_{z}\end{array}\right]^{T}$ representam, respectivamente, os deslocamentos das fases sólida e fluido. $\mathbf{H}_{s} \mathrm{e} \mathbf{H}_{f}$ são, respectivamente, as matrizes de coeficientes elásticos das fases sólida e fluído, e $\mathbf{H}_{s f}$ é a matriz de acoplamento entre as duas fases. L é a matriz contendo os operados de derivação espacial. Estas expressões são definidas, respectivamente, como segue:

$$
\begin{gathered}
\mathbf{H}_{s}=\left[\begin{array}{cccccc}
H_{11} & H_{12} & H_{13} & 0 & 0 & 0 \\
H_{12} & H_{22} & H_{23} & 0 & 0 & 0 \\
H_{12} & H_{23} & H_{33} & 0 & 0 & 0 \\
0 & 0 & 0 & G_{44} & 0 & 0 \\
0 & 0 & 0 & 0 & G_{55} & 0 \\
0 & 0 & 0 & 0 & 0 & G_{66}
\end{array}\right] \mathbf{H}_{f}=\left[\begin{array}{cccccc}
A+2 N & A & A & 0 & 0 & 0 \\
A & A+2 N & A & 0 & 0 & 0 \\
A & A & A+2 N & 0 & 0 & 0 \\
0 & 0 & 0 & N & 0 & 0 \\
0 & 0 & 0 & 0 & N & 0 \\
0 & 0 & 0 & 0 & 0 & N
\end{array}\right] \\
\mathbf{L}^{T}=\left[\begin{array}{cccccc}
\partial / \partial x & 0 & 0 & \partial / \partial y & 0 & \partial / \partial z \\
0 & \partial / \partial y & 0 & \partial / \partial x & \partial / \partial z & 0 \\
0 & 0 & \partial / \partial z & 0 & \partial / \partial y & \partial / \partial x
\end{array}\right]
\end{gathered}
$$

onde $H_{i j}(i, j=1, \ldots, 6)$ são os coeficientes elásticos da fase sólida, e $A$ e $N$ designam os coeficientes de Lamé, expressos, respectivamente, em função do módulo de Young, do coeficiente de Poisson do material, e dos módulos de compressão do material do esqueleto e do material poroso. É importante salientar que na formulação do elemento finito 
poroelástico são também considerados termos de dissipação e forças de superfície ao nível elementar conforme detalhado por Dauchez et al. (1998a).

A discretização das expressões das energias é feita considerando que o elemento poroelástico é representado por um domínio tridimensional $\Omega$ como ilustrado na Fig. 2, é formado por um conjunto de elementos de volume $\Omega_{e}$.

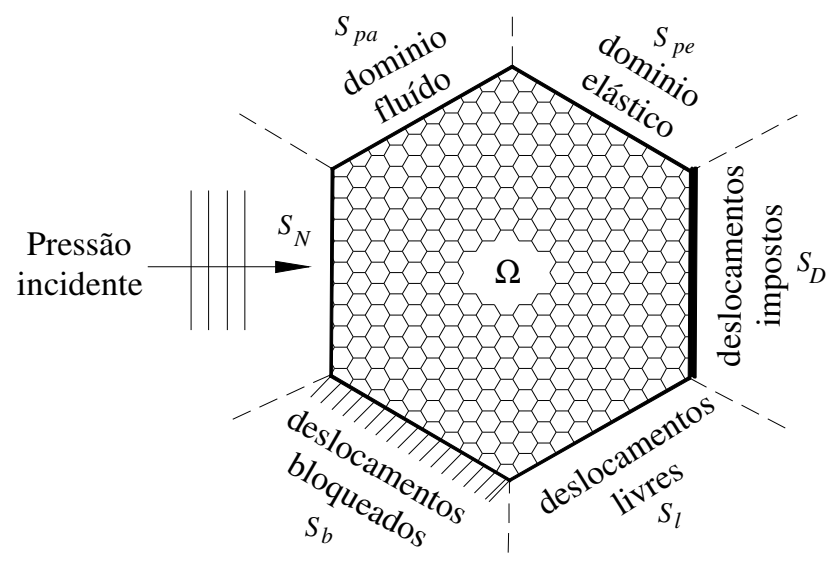

Figura 2. Discretização e condições de contorno do domínio poroelástico (adaptado de Dauchez et al, 1998).

De acordo com a discretização ilustrada na Fig. 2, o elemento poroelástico pode estar em contato com um meio fluído (ar) através da interface $S_{p a}$, e com um meio elástico (neste caso, a estrutura composta) através da interface $S_{p e}$. Além disso, ele pode ser excitado diretamente por uma onda acústica incidente expressa por uma restrição (condições de Neumann) sobre a superfície $S_{N}$, ou por uma força mecânica, expressa por um deslocamento imposto (condições de Dirichlet) sobre a superfície $S_{D}$. Deve-se ainda ressaltar que diferentes condições de limite podem ser aplicadas através da consideração de que alguns graus-de-liberdade estão livres (superfície $S_{l}$ ) e outros estão bloqueados (superfície $S_{h}$ ).

Neste trabalho, será utilizado o mesmo elemento finito plano formado por 8 nós da família serendipity mostrado na Fig. 1, cujas funções de interpolação são formuladas no sistema de coordenadas global (de Lima et al.; 2010). Portanto, as energias podem ser formuladas como segue:

$$
\begin{aligned}
& V=\sum_{\mathrm{e}} \int_{\Omega_{\mathrm{e}}} V^{(\mathrm{e})} d \Omega \\
& T=\sum_{\mathrm{e}} \int_{\Omega_{\mathrm{e}}} T^{(\mathrm{e})} d \Omega
\end{aligned}
$$

Neste sentido, através das expressões (9) e (10), as energias potencial e cinética do elemento poroelástico podem ser obtidas como segue:

$$
\begin{gathered}
V=\frac{1}{2}\left[\overline{\mathbf{u}}^{T} \mathbf{K}_{s} \mathbf{L} \overline{\mathbf{u}}+2 \operatorname{Re}\left(\overline{\mathbf{u}}^{T}(1-\phi) K_{e}(\omega) \hat{\mathbf{K}} \overline{\mathbf{U}}\right)+\overline{\mathbf{U}}^{T} \phi K_{e}(\omega) \hat{\mathbf{K}} \overline{\mathbf{U}}\right] \\
T=\frac{1}{2}\left[\rho_{11} \dot{\overline{\mathbf{u}}}^{T} \hat{\mathbf{M}}_{s} \dot{\overline{\mathbf{u}}}+2 \rho_{12} \operatorname{Re}\left(\dot{\overline{\mathbf{u}}}^{T} \hat{\mathbf{M}} \dot{\overline{\mathbf{U}}}\right)+\rho_{22} \dot{\overline{\mathbf{U}}}^{T} \hat{\mathbf{M}} \overline{\mathbf{U}}\right]
\end{gathered}
$$

onde $K_{e}(\omega)$ representa o módulo de incompressibilidade equivalente do fluido, $\phi$ é a porosidade, $\mathbf{K}_{s}$ é a matriz de rigidez da fase sólida; $\hat{\mathbf{K}}$ é a matriz de rigidez adimensional da fase fluido e acoplamento, e $\hat{\mathbf{M}}$ é a matriz de massa adimensional das fases sólida e fluido.

Portanto, as equações de Lagrange podem ser aplicadas para o sistema constituído de $n$ variáveis generalizadas $q_{i}(=1, \ldots, n)$ :

$$
\frac{\partial V}{\partial q_{i}}+\frac{d \partial}{d t}\left(\frac{\partial T}{\partial \dot{q}_{i}}\right)+\frac{\partial D}{\partial \dot{q}_{i}}=F_{i}
$$

onde $F_{i}$ representa os esforços externos. No regime harmônico de frequência $\omega$, as equações de Lagrange são reescritas sob a seguinte forma matricial: 


$$
\mathbf{Z}_{p}(\omega)\left\{\begin{array}{l}
\mathbf{u} \\
\mathbf{U}
\end{array}\right\}=\left\{\begin{array}{l}
\mathbf{F}_{s} \\
\mathbf{F}_{f}
\end{array}\right\}
$$

onde a matriz de impedância, $\mathbf{Z}_{p}(\omega)$, é composta de termos de rigidez, de massa, e de amortecimento:

$$
\mathbf{Z}_{p}(\omega)=\left[\begin{array}{cc}
\mathbf{K}_{s} & (1-\phi) K_{e}(\omega) \hat{\mathbf{K}} \\
(1-\phi) K_{e}(\omega) \hat{\mathbf{K}}^{T} & \phi K_{e}(\omega) \hat{\mathbf{K}}
\end{array}\right]-\omega^{2}\left[\begin{array}{cc}
\rho_{11} \hat{\mathbf{M}} & \rho_{12} \hat{\mathbf{M}} \\
\rho_{11} \hat{\mathbf{M}}^{T} & \rho_{22} \hat{\mathbf{M}}
\end{array}\right]+j \omega b(\omega)\left[\begin{array}{cc}
\hat{\mathbf{M}} & -\hat{\mathbf{M}} \\
-\hat{\mathbf{M}}^{T} & \hat{\mathbf{M}}
\end{array}\right]
$$

ou ainda sobre a seguinte forma:

$$
\mathbf{Z}_{p}(\omega)=\mathbf{K}_{p}-\omega^{2} \mathbf{M}_{p}+j \omega \mathbf{C}_{p}
$$

onde $b(\omega)$ representa o coeficiente de amortecimento viscoso, dependente da frequência.

\subsection{Modelagem de materiais poroelásticos}

Para a situação em que uma camada de material poroelástico é colada na camada superior ou inferior do elemento finito de placa composta mostrado na Fig. 1, e considerando as impedâncias da estrutura mecânica e do elemento poroelástico conforme definido pela Eq. (17), o problema acoplado em vibroacústica pode ser colocado da seguinte forma (Panneton, 1996; 1994; Moran e Ohayon, 1992):

$$
\left[\begin{array}{cc}
\mathbf{Z}_{e} & \boldsymbol{0} \\
\mathbf{0} & \mathbf{Z}_{p}
\end{array}\right]\left\{\begin{array}{l}
\mathbf{u}_{e} \\
\mathbf{u}_{p}
\end{array}\right\}=\mathbf{F}
$$

onde $\mathbf{Z}_{e}=\mathbf{K}_{e}-\omega^{2} \mathbf{M}_{e}$ e $\mathbf{Z}_{p}$ são as impedâncias associadas à placa composta e ao elemento poroelástico, respectivamente. Além disso, é importante destacar que nas regiões onde há acoplamento direto entre as camadas da estrutura composta e o elemento poroelástico, deve-se impor a continuidade dos campos de deslocamentos.

\section{SIMULAÇÕES NUMÉRICAS PRELIMINARES}

Nesta seção são apresentados os resultados preliminares obtidos pelas simulações numéricas com o objetivo de verificar os procedimentos de modelagem e implementação computacional de estruturas compostas contendo materiais poroelásticos para fins de controle passivo de ruído. Portanto, será avaliado o aumento do amortecimento de uma estrutura composta através do emprego de materiais poroelásticos. Além disso, é importante destacar que todo o procedimento de modelagem foi desenvolvido no ambiente de programação Matlab ${ }^{\circledR}$.

Para o problema mecânico, será considerada a placa plana composta ilustrada na Fig. 3(a), onde $L_{x}=L_{y}=0,16 \mathrm{~m}$. Além da camada de material poroelástico, a placa e formada por cinco camadas unidirecionais de mesma espessura e igual a $h / 5$, onde $h=L_{x} / 128$. A Fig. 3(b) ilustra a discretização do modelo de elementos finitos composto por 64 elementos de placa plana laminada e 225 nós. São consideradas as seguintes condições de contorno para o problema: $\mathrm{u}_{0}$ $=\mathrm{w}_{0}=\psi_{\mathrm{z}}=\varsigma_{\mathrm{x}}=\varsigma_{\mathrm{z}}=0$ em y $=0$ e $\mathrm{y}=\mathrm{a}$, e $\mathrm{u}_{0}=\mathrm{w}_{0}=\psi_{\mathrm{z}}=\varsigma_{\mathrm{y}}=\varsigma_{\mathrm{z}}=0 \mathrm{em} \mathrm{x}=0$ e $\mathrm{x}=\mathrm{b}$ (Correia, 2000). Além disso, as orientações das fibras de cada camada são adotadas arbitrariamente como $\left(45^{\circ} / 0^{\circ} / 45^{\circ} / 0^{\circ} / 45^{\circ}\right)$, e a propriedades elásticas de cada camada são: $\bar{E}_{1}=172,4 \mathrm{GPa}, \bar{E}_{2}=\bar{E}_{3}=6,89 \mathrm{GPa}, \bar{G}_{12}=\bar{G}_{13}=3,45 \mathrm{GPa}, \bar{G}_{23}=1,38 \mathrm{GPa}, v_{12}=v_{13}=0,25$, $v_{23}=0,30 . \rho=1566 \mathrm{~kg} / \mathrm{m}^{3}$ é a densidade do material.

Para o material poroelástico, são consideradas as seguintes características: porosidade, $\phi=0,98$, resistividade, $\sigma=$ $0,98 \mathrm{Nm}^{-4} \mathrm{~s}$; tortuosidade, $\alpha_{\infty}=1,2$; comprimento viscoso característico, $\Lambda=220 \mu \mathrm{m}$; comprimento térmico característico, $\Lambda^{\prime}=490 \mu \mathrm{m}$; densidade equivalente, $\rho_{1}=30 \mathrm{kgm}^{-3}$, coeficiente de amortecimento, $\eta_{s}=0,12$; Poisson, $v=0,3$; e módulos de Young, $E_{1}=250000 \mathrm{~Pa}$ e $E_{2}=180000 \mathrm{~Pa}$ e. É importante destacar que estas propriedades são utilizadas para o cálculo da expressão, $\mathbf{K}_{e}(\omega)$, conforme Eq. (18) e definida segundo Dauchez et al. (1998a). 


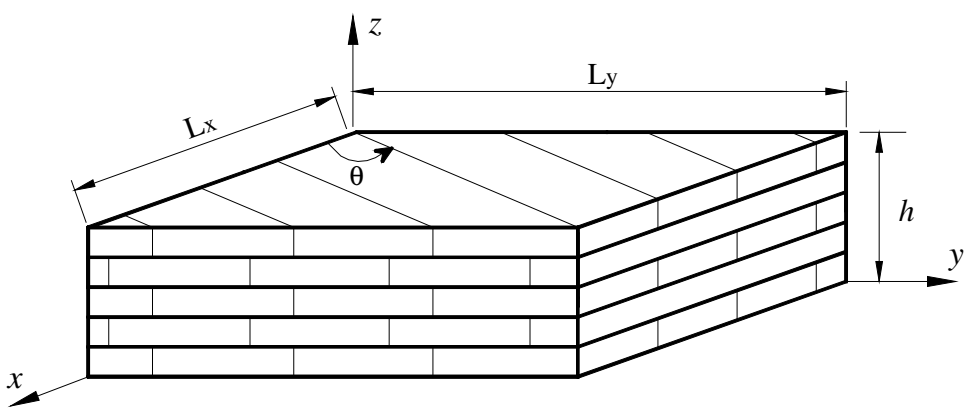

(a)

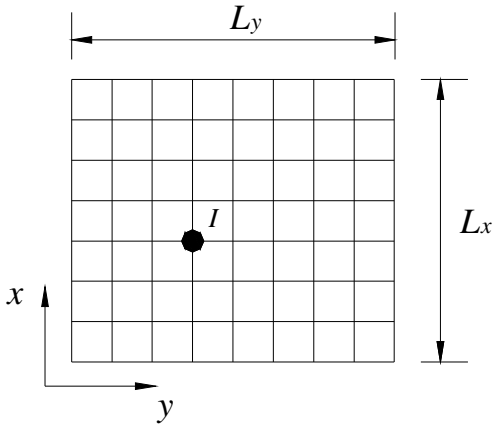

(b)

Figura 3. Geometria da placa composta com material poroelástico (a); discretização por EFs (b).

Os cálculos consistem em obter os níveis de energia vibratória da placa composta sem e com material poroelástico acoplado calculados em bandas de frequências de $1 / 3$ de oitava, para a resposta dinâmica pontual associada ao ponto $I$, escolhido arbitrariamente, e indicado na Fig. 3(b). É importante destacar que o sinal da resposta dinâmica é filtrado de forma a apenas deixar passar bandas de frequência de 1/3 de oitava, e quantificar a energia vibratória do sistema contida nessas bandas através da seguinte expressão:

$$
\left|H_{e}\left(\omega_{i}\right)\right|=\frac{1}{\left|1+7300\left(\omega_{i} / \omega_{e}\right)^{6}\right|}
$$

Onde $\left|H_{e}(\omega)\right|$ representa a resposta dinâmica do filtro, $\omega_{i}$ é a frequência do sinal, e $\omega_{c}$ é a frequência do filtro.

Portanto, a energia contida na banda de $1 / 3$ de oitava a partir da resposta dinâmica do sistema, pode ser computada como segue:

$$
P=10 \log _{10}\left(\left|\sum_{i=1}^{n f r e q}\right| H_{c}\left(\omega_{i}\right)\left|\times H\left(\omega_{i}\right)\right| / p o\right)
$$

onde $P[d B]$ é energia vibratória contida na banda de $1 / 3$ de oitava, $H\left(\omega_{i}\right)$ representa a resposta dinâmica do sistema, e o termo $p o=20 \mu \mathrm{Pa}$ representa a referência. Além disso, a Tabela 1 apresenta os índices das frequências centrais dos filtros de 1/3 de oitava, e os respectivos valores dessas frequências padronizados por normas internacionais.

Tabela 1. Frequências centrais dos filtros

\begin{tabular}{ccccc}
\hline $\mathrm{N}^{\mathrm{o}}$ Freq. & 1 & 2 & 3 & 4 \\
\hline Freq. central & 1.25 & 1.6 & 2 & 2.5 \\
\hline
\end{tabular}

A Fig. 4 a seguir mostra as amplitudes das funções de resposta em frequência computadas para a placa composta sem a presença do material poroelástico, e a Fig. 5 representa as energias em bandas de terço de oitava computadas para cada um dos modos de vibração da placa composta com material poroelástico. Através da Fig. 5 fica evidente que ocorre uma razoável atenuação dos níveis da energia vibratória do sistema acoplado na banda de frequência de interesse. Isto pode ser explicado pelo fato de que o esqueleto do material poroelástico estando em contato com a placa composta em vibração, o mesmo é solicitado, sendo que neste caso, a quantidade de dissipação estrutural passa a ser proporcional ao módulo de elasticidade e do coeficiente de amortecimento do esqueleto. Além disso, a eficiência do material poroelástico depende também da resistividade de passagem do ar e da velocidade relativa entre o fluido e o esqueleto. 


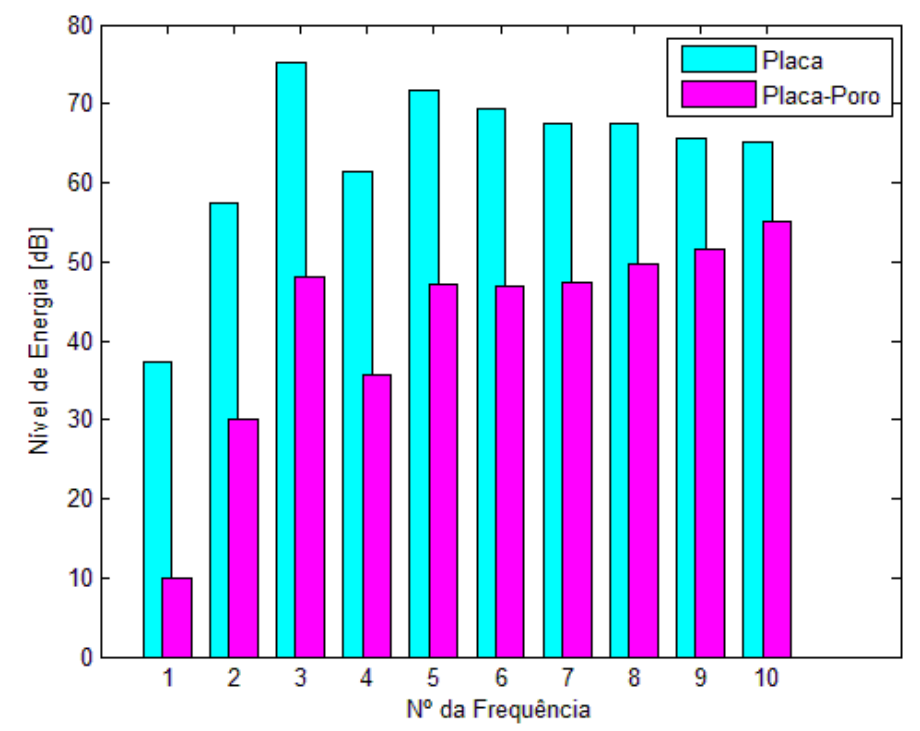

Figura 4. Energia vibratória calculada em bandas de 1/3 de oitava.

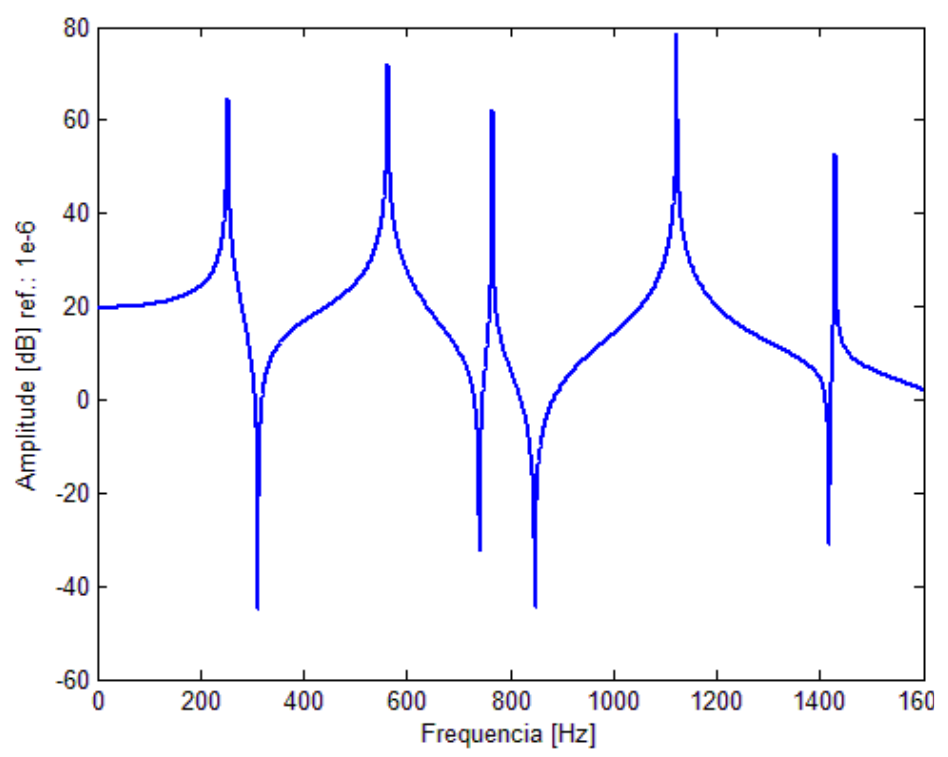

Figura 5. Amplitudes das funções de resposta em frequência do sistema sem e com material poroelástico.

\section{CONCLUSÕES GERAIS}

De acordo com estudos previamente realizados e nos resultados preliminares apresentados neste artigo, pode-se concluir que os procedimentos de modelagem desenvolvidos permitem representar o comportamento dinâmico dos materiais poroelásticos acoplados a estruturas compostas, notadamente para representar a capacidade de dissipação de energia vibratória do sistema. Além disso, deve-se destacar que foi feita uma parametrização do modelo de elementos finitos do sistema acoplado em vibroacústica que demonstrou ser bastante útil obtendo um ganho de custo computacional.

Outro aspecto importante que deve ser salientado é que foi observado que a dissipação viscosa do material poroelástico pode ser otimizada através da escolha correta de alguns parâmetros do sistema, tais como resistividade ao fluxo, uma vez que foi observado que este parâmetro exerce uma grande influência na eficiência do material poroelástico. Entretanto, cuidado deve ter com polímeros mais rígidos, sendo que neste caso, os mecanismos de dissipação são advindos na maior parte das vibrações do esqueleto.

Por fim, deve-se salientar que testes numéricos estão em curso de realização para verificação dos códigos de elementos finitos implementados e validação com outros trabalhos disponíveis na literatura.

\section{AGRADECIMENTOS}

Os autores gostariam de agradecer à Agência de Fomento à Pesquisa do Estado de Minas Gerais FAPEMIG pelo suporte financeiro de suas atividades de pesquisa através do Projeto TEC-APQ-02522-12, e a Agência de Fomento à 
Pesquisa $\mathrm{CNPq}$ pelo contínuo suporte às atividades de pesquisa dos autores envolvidos neste trabalho, especialmente através do Projeto 303020/2013-0 (A.M.G. de Lima). Por fim, os autores expressam seus agradecimentos ao INCT-EIE, financiado pelo CNPq, CAPES e FAPEMIG.

\section{REFERÊNCIAS}

Biot, M. A., 1956, "The Theory of Propagation of Elastic Waves in a Fluid-Satured Porous Solid. I-Low Frequency Range. II-High Frequency Range", J. Acoust. Soc. Am., Vol 28, pp 168-191.

Boily, S. and Charron, F., 1999, "The Vibroacoustic Response of a Cylindrical Shell Structure with Viscoelastic and Poroelastic Materials", Journal of Applied Acoustics, Vol 58, pp. 131-152.

Champoux, Y. and Allard, J. F., 1991, "Dynamic Tortuosity and Bulk Modulus in Air-Saturated Porous Media", J. Acoust. Soc. Am., Vol 89, pp 910-916.

Christensen, R. M., 1982, “Theory of viscoelasticity: An introduction”, Academic Press, Inc., New York, 2nd edition.

Clark, R.L., Füller, C.R., 1992, "Modal sensing of efficient acoustic radiators with polyvinylidene fluoride distributed sensors in active structural acoustic control approaches", Journal of the Acoustical Society of America, vol. 91, pp. 3321-3329.

Dauchez, N., Sahraoui, S., Atalla, N., 1998a, "Vibroacoustic Behavior of a Porous Layer Bonded onto a Plate", $4^{\text {th }}$ AIAA/CEAS Aeroacoustics Conference, Toulouse, France.

Dauchez, N., Sahraoui, S., Atalla, N., 1998b, "Numerical Analysis of Damping Added by a Foam Layer on a Vibrating Plate", $16^{\text {th }}$ ICA and $135^{\text {th }}$ Meeting of the ASA, Seattle, Washington, USA.

Delany, M. E. and Bazley, E. N., 1970, “Acoustical Properties of Fibrous Materials”, Applied Acoustics, Vol 3, pp. 105116.

Johansen, T. F., Allard, J. F., Brouard, B., 1995, "Finite Element Method for Predicting the Acoustical Properties of Porous Samples", Acta Acoustica, Vol 3, pp. 487-491.

Johnson, D. L., Koplik, J., Dashen, R., 1987, "Theory of Dynamic Permeability and Tortuosity in Fluid-Saturated Porous Media”, J. Fluid Mechanics, Vol 176, pp. 379-402.

Lima Jr., J. J. de, Arruda, J. R. F., 1997, "Finite Element Modeling of Piezoceramic Sensors and Actuators", Anais do XVIII CILANCE, Congresso Ibero Latino - Americano de Métodos Computacionais para Engenharia, Brasília, v1, pp. 751-757.

Moran, H. J. P. and Ohayon, R., 1992, Interactions fluide-structures, Paris, Masson.

Panneton, R., Atalla, N., 1994, “A 3-D Finite Element Model for Sound Transmission Through a Double-Plate System with Isotropic Elastic Material”, $128^{\text {th }}$ Meeting of Acoust. Soc. Am., J. Acoust. Soc. Am., Vol 96, pp. 3339 (A).

Panneton, R., Atalla, N., 1996, "Numerical Prediction of Sound Transmission Through Finite Multilayer Systems with Poroelastic Materials", J. Acoust. Soc. Am., Vol 100, pp. 346-354.

\section{RESPONSABILIDADE AUTORAL}

“Os autores são os únicos responsáveis pelo conteúdo deste trabalho”. 


\title{
FINITE ELEMENT MODELING OF COMPOSITE STRUCTURES INCORPORATING POROELASTIC MATERIALS
}

\author{
Antônio Marcos Gonçalves de Lima, amglima@ mecanica.ufu.br \\ Victor Augusto da Costa Silva, Victor_augusto_cn@hotmail.com
}

Federal University of Uberlândia, School of Mechanical Engineering, Campus Sta Mônica, Uberlândia-MG, Brazil Federal University of Uberlândia, School of Mechanical Engineering, Campus Sta Mônica, Uberlândia-MG, Brazil

\begin{abstract}
The study of dynamic behavior of composite structures incorporating poroelastic materials for vibration and noise attenuations is motivated by their application in the context of real-word engineering structures. In this context, this work is dedicated to the numerical and computational studies of composite plate structures with poroelastic materials to be applied as surface treatments to form the sandwich plate system. In order to model the dynamic behavior of the poroelastic materials, the generalized theory originally proposed by Biot is retained herein. For the structural composite elements incorporating poroelastic materials the equivalent layer theory combined with the firstorder shear deformation theory will be used. The preliminary numerical results confirm the interest in using the poroelastic materials to reduce the noise levels of the system subjected to an external excitation.
\end{abstract}

Keywords: Composite structures, poroelastic materials, passive control, finite elements. 